Crises de l'art moderne latino-américain, 1920-1980 : le rôle de Marta Traba dans la rupture et la recherche d'un nouvel ordre artistique

\title{
Elsa Crousier
}

\section{(2) OpenEdition} Journals

Édition électronique

URL : https://journals.openedition.org/cher/3757

DOI : 10.4000/cher.3757

ISSN : 2803-5992

Éditeur

Presses universitaires de Strasbourg

\section{Édition imprimée}

Date de publication : 1 décembre 2015

Pagination : 209-219

ISBN : 978-2-86820-913-9

ISSN : 1968-035X

Référence électronique

Elsa Crousier, «Crises de l'art moderne latino-américain, 1920-1980 : le rôle de Marta Traba dans la rupture et la recherche d'un nouvel ordre artistique », reCHERches [En ligne], 15 | 2015, mis en ligne le 01 décembre 2021, consulté le 14 décembre 2021. URL : http://journals.openedition.org/cher/3757 ; DOI : https://doi.org/10.4000/cher.3757 


\title{
Crises de l'art moderne latino-américain, 1920-1980: le rôle de Marta Traba dans la rupture et la recherche d'un nouvel ordre artistique
}

\author{
ELSA CROUSIER \\ Université Lumière Lyon 2 - LCE
}

De alguna manera, mi propósito es hacer un análisis, más que inductor, provocador.

Decirle al público: estas imágenes son suyas, poséalas.

Lo representan: cúbrase con ellas como los brujos con sus mantos.

Lo explican por encima de las apariencias : tenga fe en esta explicación simbólica.

(Traba 1975:8)

«A nueva sociedad, pintura nueva » prônait José Martí (Rodríguez Prampolini 1997:139), au moment où l'Amérique latine se battait pour ses dernières indépendances politiques et allait ainsi mettre un terme à plus de trois siècles de colonisation politique, économique et culturelle. Toutefois, cette «nouvelle peinture» n'est pas allée de pair avec les indépendances. La longue période de domination culturelle qu'a connue le sous-continent a eu des conséquences durables sur sa production artistique ${ }^{1}$ : les artistes nont pas immédiatement recherché une expression artistique proprement latino-américaine. En cherchant à séloigner de l'héritage ibérique, qui évoquait par trop la période de la colonisation, ils ont créé un autre type de dépendance, avec d'autres pays européens, comme la France ou l'Allemagne, puis nord-américains. Dans les arts plastiques, bien plus longtemps qu'en littérature, l'Europe était, au XIX ${ }^{e}$ siècle et jusqu'aux années 1920, le modèle à suivre pour la plupart des artistes (Bayón

1 Cette étude a pour objet l'art moderne latino-américain à portée nationale ou internationale. Nous ne pourrons donc pas y analyser les manifestations culturelles populaires (elles ont toutefois une influence indéniable sur la production artistique nationale). 
1980 : 5-33). Marta Traba (1923-1983), figure pionnière de la critique d'art latinoaméricaine, soulignait l'origine ambivalente de ce phénomène qui relevait certes de la «dominación cultural francesa y europea» (Traba 1972: 13), mais était également due à une "sumisión» (Traba 1961: 43) volontaire des artistes aux canons artistiques étrangers ${ }^{2}$.

Ces éléments de contexte éclairent deux périodes de crises successives dans l'art latino-américain du $\mathrm{xx}^{\mathrm{e}}$ siècle. La première commence dans les années 1920 et témoigne des naissances parallèles d'une «conscience latino-américaine» et de l'art moderne en Amérique latine: les artistes accordent une importance croissante à une expression artistique libre, qui serait mieux à même d'incarner l'identité culturelle latino-américaine ${ }^{3}$. Cette prise de conscience conduit à une profonde remise en cause des principes de création artistique. La deuxième crise éclate quant à elle entre les années 1960 et 1980, en réaction à ce qui est perçu par la critique comme un nouveau type de «coloniaje estético» (Traba 2005: 80), celui des États-Unis et du pop'art, qui «envahit» les sociétés du sous-continent et en bouleverse les normes esthétiques.

Nous nous proposons donc d'interroger les manifestations de ces deux crises de l'art latino-américain et le rôle qu’a joué le discours critique, en particulier celui de Marta Traba, dans la compréhension de ces crises. Dans quelle mesure l'analyse critique de Marta Traba concernant la première crise lui permet-elle de porter un regard pertinent sur la deuxième crise et d'ouvrir la voie, au-delà du renversement des normes établies, à de nouveaux principes créatifs?

\section{Un art latino-américain?}

À partir des années 1920, parallèlement à l'apparition de l'art moderne en Amérique latine, naît dans les arts plastiques une conscience de la spécificité latino-américaine que les artistes cherchent à exprimer dans leurs ouvres, en renversant les principes de création esthétique en vigueur. Il s'agit là d'un prolongement de la question identitaire: «¿quiénes somos?» (Bayón 1980: 20), déjà fort présente depuis la fin du xix siècle chez nombre d'historiens ou écrivains latino-américains. Cette définition de soi se fait généralement par assimilation ou opposition à un "autre» (qui a d'abord été l'Européen), et les réponses qui ont été apportées à ce questionnement correspondent aux deux principales tendances de l'art moderne latino-américain: absorber des théories

2 D’après Marta Traba, si l'implantation des Académies européennes a favorisé cette situation dans divers pays latino-américains, les peintres du sous-continent ont aussi bien souvent leur part de responsabilité dans cette «colonisation» car nombre d'entre eux allaient se former en Europe et se soumettaient aux canons et mouvements artistiques étrangers.

3 Nous entendons le concept d'«identité culturelle» dans les arts plastiques comme la recherche menée par les artistes pour trouver une expression propre, sans appliquer des canons artistiques venus d'ailleurs, pour résister et répondre aux diverses propositions ou impositions culturelles venues de l'étranger. 
et des mouvements étrangers, ou trouver des voies artistiques propres afin de parvenir à une expression latino-américaine.

Dans les années 1970, dans un contexte intellectuel où croît l'intérêt pour la question des identités culturelles, Ángel Rama développe le concept de «transculturation $»^{4}$. Le critique littéraire uruguayen étudie la narration latino-américaine à l'aune de l'anthropologie, la sociologie et la philosophie, et considère que l'identité culturelle latino-américaine serait le résultat d'une succession de «transculturations». Cette théorie est élaborée pour étudier la production littéraire du sous-continent, mais elle est à nos yeux une grille d'analyse également pertinente pour comprendre l'histoire des arts picturaux, et en particulier les divers modes latino-américains d'appropriation artistique.

Pour les théoriciens et les artistes qui cherchent à frayer la voie d'un art proprement latino-américain, deux problèmes se posent: que représenter et comment le représenter? D’abord, l'œuvre d'art ne peut se limiter à exprimer une idée politique ou véhiculer une idéologie; si elle ne participe pas d'une recherche formelle ou esthétique, elle ne saurait être considérée comme une véritable création artistique: "Todo catecismo político, aun el mejor ante los mejores, es un disco, un cliché, una cosa muerta, ante la sensibilidad del artista" (César Vallejo, cité par Traba 1973: 298). Si l’art latino-américain est nécessairement lié au contexte de sa création, il ne lui est pas réductible, et il est seulement un terreau sur lequel l'artiste crée librement.

Le second enjeu est celui du médium et du style: comme le montre Hans Belting, l'image se compose d'un côté de l'objet, l'idée ou le concept représenté, et de l'autre du médium ${ }^{5}$. Toutes les images qui se forment dans notre société, le font "par l'entremise des médiums qui leur confèrent la visibilité " (Belting 2004: 7). Or jusque dans les années 1920, l'art du sous-continent ne s'exprime que très peu par un médium propre, car pour représenter des sujets latinoaméricains (paysages, coutumes, histoire...) les artistes «importent» en général leurs supports et leurs styles. Aux yeux des critiques d'art des années 1960-1970, l'émancipation de l'art en Amérique latine semble alors liée à l'élaboration d'un médium et d'un style propres.

Ce sont là les problématiques majeures qui innervent la «prise de conscience latino-américaine» des artistes du début du $\mathrm{xx}^{\mathrm{e}}$ siècle, et qui permettent d'en éclairer les démarches esthétiques.

4 La «transculturation» est l'adaptation à des impositions culturelles venues de l'extérieur, suivant un processus de pertes, de sélections, de redécouvertes et d'incorporations artistiques. Ángel Rama reprend et adapte à la littérature le concept de «transculturación» développé par F. Ortiz (Rama 1987: 32-33).

5 Ce concept désigne les moyens historiquement déterminés pour créer et recréer des images (tableaux, films, outils, etc., dans lesquels les images s'incarnent et apparaissent). 


\section{La première crise de l'art latino-américain (1920-1950): un renversement des normes}

«Antes de 1900 [...] pesaba aún sobre los artistas la imposición física de la colonia cuyas prolongaciones en la vida cultural no terminan con la independencia» (Traba 1961: 13-14). À la fin du xIX ${ }^{e}$ siècle, la production artistique connaît effectivement plusieurs types d'influence européenne: non seulement des artistes européens viennent peindre l'Amérique, comme le Français Raymond Monvoisin (1790-1870) ou l'Allemand Ernesto Kirchbach (18321880), mais ces mêmes peintres étrangers sont souvent fondateurs ou directeurs d'Académies latino-américaines, et ils ont à ce titre une autorité institutionnelle sur la production artistique. Si certains artistes s'intéressent et se consacrent à des sujets latino-américains, leurs styles picturaux restent souvent conformes aux styles européens. On observe cette tendance aussi bien dans le paysage que dans la peinture de genre ou «sociale», ces dernières étant influencées en particulier par Millet, comme le montre l'œuvre d'Arturo Michelena (1863-1898).

Lémancipation culturelle latino-américaine se comprend en lien avec la naissance de l'art moderne en Amérique latine. Il permet une plus grande liberté formelle et autorise progressivement les artistes à séloigner de la norme européenne. Cependant, il est apparu dans le sous-continent bien plus tard qu'en Europe et aux États-Unis: tandis que la première exposition impressionniste avait lieu en 1874 à Paris, on situe les débuts de l'art moderne en Amérique latine autour des années $1920^{6}$. C'est là une période charnière où s'effectue un renversement, une mise en cause des directives et institutions artistiques européennes. Cependant, il ne s'agit au début que de recherches individuelles.

L'impressionnisme est le premier mouvement qui ait eu un fort impact sur la conscience des artistes latino-américains: si certains peintres suivent fidèlement les codes impressionnistes pour représenter des réalités locales, comme le paysagiste Fernando Fader (1882-1935), d'autres y trouvent le moyen d'une libération formelle. Juan Francisco González (1853-1933), s'inspirant des principes de l'impressionnisme, joue avec la texture de la peinture à l'huile pour capter l'atmosphère, mais il ne décompose pas les couleurs avec la rigueur caractéristique des impressionnistes. Ces techniques apparaissent dans son tableau Calle de San Fernando (1921): le coup de pinceau y est vigoureux, le peintre utilise des couleurs pures et concentre la lumière dans certaines zones. Dans le portrait Mariana (1926), il inverse le profil classique et travaille sur la texture de la peinture, lui donnant un aspect d'aquarelle. Il est intéressant de constater que Marta Traba le considère comme "libremente impresionista" (Traba 1994: 70), suggérant par là qu'une appropriation stylistique se conçoit avant tout comme une émancipation.

6 Ces phénomènes sont analysés dans les ouvrages d'histoire de l'art de Damián Bayón (1980: 5-6 et 19) et de Marta Traba (1961: 14 et 1994:3). 
Le début de crise identitaire liée à la naissance de la peinture moderne ne se manifeste pas seulement dans des initiatives personnelles. Cette nouvelle préoccupation apparaît aussi dans certains mouvements d'ampleur nationale, dont le mot d'ordre est le renversement de la norme établie: c'est le cas du muralisme mexicain. Il s'agit du premier mouvement artistique latino-américain mondialement reconnu comme tel. Ses principaux artistes, comme Diego Rivera (1886-1957) ou David Alfaro Siqueiros (1896-1974), représentent des épisodes de l'histoire du Mexique ou des hymnes à la révolution et à la liberté. Mais le muralisme est ambivalent d'un point de vue esthétique, car si le médium utilisé - la fresque murale - est original, ce projet reste formellement très proche des styles occidentaux (comme l'expressionnisme), et, en portant avant tout un message révolutionnaire, il court le risque de s'y voir borné.

Le mouvement anthropophage au Brésil (1924-1928) va plus loin dans la recherche d'une expression latino-américaine. Sa démarche est ainsi définie dans le Manifeste Pau-Brasil (1924) puis le Manifeste Anthropophage (1928) comme «la théorie de l'avalement critique d'un legs culturel universel [...] développée depuis le point de vue inflexible d'un "mauvais sauvage", celui qui mange les hommes blancs, le cannibale ${ }^{7}$. C'est la revendication d'une «transculturation» par le cannibalisme: le cannibale, en dévorant les cultures, les digère et se les approprie. L'œuvre Abaporu (1928) de Tarsila do Amaral (1886-1973) illustre bien le mot d'ordre et l'originalité formelle du mouvement: elle représente un immense corps nu, qui s'intègre comme un élément naturel au paysage dans lequel il se repose. Son titre, «l'homme qui mange les personnes», est donné en langue tupi, et ce choix lui-même est significatif, les Tupis étant des Amérindiens anthropophages du Brésil lors de la colonisation. De même, la Escuela del Sur en Uruguay (1935), mouvement conduit par Joaquín Torres García (18741949), cherche à donner forme à un art américain à partir d'une opération de décontextualisation et de resémantisation. Le croquis de Torres García, Nuestro norte es el Sur (1936), qui représente une carte de l'Amérique latine inversée, symbolise de façon expressive la crise identitaire. Il invite le spectateur à se demander pourquoi l'hémisphère nord devrait être le seul "pôle» artistique. Ce croquis montre que la crise est née de la possibilité de renverser la norme, en inversant les références: nous vivons sur une sphère, il n'y a donc pas de haut ou de bas, ce ne sont que des conventions établies par les premières puissances coloniales. Le pôle sud pourrait donc être le nouveau nord, et la constellation de la croix du sud remplacerait alors la grande ourse, indiquant aux artistes la voie à suivre pour construire leurs propres normes esthétiques.

Enfin, l'arrivée du cubisme en Europe a une importance fondamentale pour les artistes latino-américains jusqu'aux années 1950-1960. Wifredo Lam, Emilio Pettoruti, Rufino Tamayo ou encore Candido Portinari produisent des œuvres

7 Haroldo de Campos cité par A. Giunta: «[...] the theory of a critical swallowing of a universal cultural legacy, developed [...] from the uncompromising viewpoint of the "bad savage", the one that eats white men, the cannibal» (Mosquera 1955: 57). 
marquées par un style personnel et de nombreuses «transculturations». Tandis que les peintres cubistes européens s'inspiraient des formes et des structures de l'art primitif africain, donc "exotique», un artiste comme Wifredo Lam (1902-1982) propose une réappropriation du cubisme en combinant la culture métissée qui est la sienne, où convergent des éléments formels et symboliques tribaux, et des inspirations surréalistes issues de la tradition latino-américaine de valorisation de l'irrationnel, plutôt que des réflexions théoriques européennes. Mythes et syncrétisme religieux nourrissent ses œuvres qui séloignent de l'intellectualisme européen. Progressivement, toute une génération d'artistes explore de nouvelles voies esthétiques et transforme, adapte et invente les styles picturaux du sous-continent.

\section{Le rôle de Marta Traba, critique d'art: théorisation et divulgation du renversement}

La production de critique et d'historienne de l'art de Marta Traba comporte deux moments bien distincts. Lorsquelle arrive en Colombie en 1954, Marta Traba entreprend de sensibiliser un large public colombien à un art moderne centré sur les mouvements européens. Mais c'est la seconde étape de sa critique d'art, que l'on situe généralement au début des années $1960^{\circ}$, qui retiendra notre attention ici: Marta Traba prend fait et cause pour les artistes dont les œuvres incarnent et valorisent l'identité latino-américaine, en recourant à une expression artistique moderne, libre, non conventionnelle?

Dans un premier temps, Marta Traba propose une lecture théorique de la crise de l'art moderne latino-américain sous deux formes complémentaires. En 1961, elle écrit La pintura nueva en Latinoamérica, l'une des premières

8 Les diverses analyses réalisées sur la production critique de Marta Traba situent cette évolution théorique entre 1960 et 1965: Rubén Darío Flórez Arcila évoque un changement dans les années 1959-1960 directement lié à sa participation à la revue Semana (Zalamea 2010: 47-64); Florencia Bazzano et Beatriz González, situent l'évolution de sa posture face au pop'art en 1964 (Bazzano 2006: 22-23); enfin, Beatriz González souligne l'influence décisive du «XVII Salón de Artistas Colombianos», en 1965, sur cette nouvelle position (Zalamea 2010: 69-77).

9 Le rôle de la critique d'art tel que nous l'entendons dans cette analyse est très varié: il consiste, certes, à recenser des phénomènes, à les analyser et à les juger, mais aussi, grâce à ce travail, à former un public, à rationaliser la valorisation et le goût en formulant des instruments conceptuels d'analyse, comme le mentionne Efrén Giraldo. Ces fonctions font du critique d'art un historien de l'art, un critique, un théoricien, mais aussi un formateur et un pédagogue. Autant de fonctions que les critiques d'art latino-américains avaient très peu assumées jusqu'aux années 1950 : dans la première moitié du $\mathrm{xx}^{\mathrm{e}}$ siècle, la critique était davantage une "conscience artistique» qu'un véritable champ d'étude (Pizarro 2002: 116). La nouvelle génération de critiques à laquelle appartient Marta Traba a donc entrepris une révision de la critique d'art et a signalé ses diverses carences. C'est parce que son ouvre comprend l'ensemble de ces caractéristiques que Marta Traba est considérée comme l'une des premières critiques d'art en Amérique latine (Giraldo 2007:9-16). 
histoires de l'art s'intéressant à l'Amérique latine dans son ensemble ${ }^{10}$. Elle analyse, dans cet ouvrage et les suivants, les différentes réactions des artistes face aux mouvements venus de l'extérieur, en insistant sur les nouvelles solutions picturales et sur la possibilité d'un art proprement américain. Comme le fait remarquer le critique d'art Juan Acha, "por primera vez se enfocó el arte de nuestros países como totalidad y con un espíritu latinoamericanista [...] con una conciencia ávida de reconocer su identidad cultural mediante la búsqueda de soluciones a los problemas artísticos y sensitivos que nos son comunes » (Giraldo 2007 : 98). En tant qu'historienne de l'art, elle est l'une des premières à témoigner des profondes mutations qui s'opèrent dans l'art latino-américain. Non seulement elle les observe et les recense, mais, en leur donnant un cadre théorique, elle les rend plus conscientes et contribue à leur vitalité. Elle s'appuie sur des valeurs qui répondent à ces changements, sans pour autant limiter son discours à une revendication identitaire: "La captación de la estructura, del condicionamiento social o del factor de época no impide juicios sobre las características del artista como categoría de análisis, del estilo como resumen de la individualidad creadora y del lienzo social como fondo para el acto del sujeto cultural» (Giraldo 2007: 84).

Marta Traba donne alors une image concrète de ce processus de crise artistique en établissant une carte des «zones artistiques» d'Amérique latine pour les années 1950-196011. Elle distingue ainsi deux grandes tendances artistiques, qui correspondent à des «zones» géographiques délimitées. Dansles «zones fermées ", la création picturale parvient à conserver une identité propre à chaque région latino-américaine tout en s'adaptant à la modernité. Cette préservation est intimement liée aux caractéristiques socio-économiques de ces zones, comme la limitation des échanges avec l'extérieur ou le poids de la tradition (il s'agit principalement de pays comme le Pérou, la Colombie, la Bolivie, etc.). En revanche, les «zones ouvertes» assimilent les modèles européens et considèrent que la perte de l'identité favorise l'entrée de l'Amérique dans le champ de l'art moderne. Il s'agit principalement de capitales de pays qui connaissent de forts échanges avec l'étranger: Buenos Aires, Caracas, Sao Paolo, entre autres. Marta Traba qualifie les zones fermées de "zones résistantes», c'est-à-dire résistant à la norme culturelle qui leur était imposée jusque-là, et encourage cette posture (Traba 1995: 79-139).

L'ensemble de la carrière de Marta Traba est marqué par un désir de diffusion et de formation du public, condition nécessaire à un véritable renversement des valeurs à l'échelle de la société. Déjà, le premier pan de sa production critique avait connu une diffusion d'ampleur nationale en Colombie grâce à une présence médiatique inédite: Marta Traba présente plusieurs programmes télévisés

10 Auparavant, Ángel Guido avait publié en 1944, Redescubrimiento de América en el arte.

11 Principalement, dans ses ouvrages publiés entre 1961 et 1973, où elle emploie les termes «áreas abiertas» et «áreas cerradas». 
d'histoire de l'art (principalement de 1954 à 1959) ${ }^{12}$, assure de nombreux cours dans les grandes universités de Bogotá (América, Los Andes et Nacional) et fonde en 1963 le Musée d’art Moderne de Bogotá. Par conséquent, dans un second temps, afin que ses théories artistiques défendant les identités culturelles latino-américaines atteignent un public plus large, Marta Traba séloigne quelque peu de la Colombie, pour, cette fois-ci, diffuser sa critique dans l'ensemble du continent sous des formes variées: une abondante publication de monographies et d'articles (au Venezuela, au Mexique, à Porto Rico, aux États-Unis), et l'enseignement dans de nombreuses universités (dans divers pays d'Amérique latine et aux États-Unis). C’est en étant consciente des enjeux de transmission et de vulgarisation des théories de l'art que Marta Traba a pu faire connaître les artistes latino-américains et contribuer à transmettre largement de nouvelles valeurs esthétiques.

\section{Comprendre et réagir face à la deuxième crise}

Le paysage artistique latino-américain tel qu'il est théorisé par Marta Traba connaît une deuxième transformation radicale dans les années 1960-1970. Marta Traba considère l'arrivée du pop'art en Amérique latine comme un nouveau "coloniaje estético», car les artistes latino-américains adoptent ce style sans le questionner. Elle réutilise alors les grilles d’analyse qu’elle avait forgées lors de la première crise artistique pour caractériser cette nouvelle colonisation esthétique, proposer une déconstruction et, enfin, envisager une alternative.

Marta Traba souligne les différences entre cette situation de crise liée au pop'art et la première crise latino-américaine: le pop'art qui naît aux États-Unis dans les années 1960 n'est pas seulement un style artistique, il est aussi un style de vie, l'esprit d'une génération, comme l'indique Tilman Osterwold: « La culture pop et le mode de vie des années soixante sont intimement liés l'un à l'autre» (Osterwold 2011: 6). En outre, c'est une manifestation culturelle essentiellement nord-américaine, née dans une société libérale, de consommation, industrielle et technologique, marquée par l'apparition des mass media. Pour Marta Traba, ces éléments socio-économiques expliquent la nature d'un nouveau langage artistique: «la actividad artística deriva directamente de las alternativas de la sociedad de consumo [...] resultando [...] tanto más alienada por su propia sociedad cuanto más proclama su libertad» (Traba 2005: 58). Elle souligne, en reprenant les études de Marcuse, que la technologie produit un «lenguaje ritual autoritario» (Traba 2005: 59) et que l'art qui naît de ce contexte en véhicule donc l'idéologie technocratique et consumériste.

12 Il s'agit de la période de plus grande influence télévisuelle de Marta Traba. D’autres diffusions ou rediffusions ont lieu ultérieurement, mais le programme «Puntos de vista» (1966) est suspendu en septembre 1966 pour des raisons politiques. Ce n'est qu'en 1983 que Marta Traba enregistre à nouveau un programme d'histoire de l'art pour la télévision colombienne, "Historia del arte moderno contada desde Bogotá», mais il n'est diffusé qu'en 1984, après sa mort (Gómez Echeverri 2008: 19-29). 
Or, les artistes latino-américains adoptent si rapidement ce nouveau style que Marta Traba parle de «década de la entrega» (Traba 2005: 141-204). Pour elle, il n’y a pas là «transculturation», mais imposition d'une civilisation. Au lieu d'importer des styles ou des éléments formels, l'Amérique latine est soumise à l'influence d'un système culturel global, que Marta Traba appelle «système de civilisation", dont la diffusion est en fait une expansion impérialiste (Traba 2005: 61). La "transculturation» ne peut donc pas avoir lieu. Aux yeux de Marta Traba, la société latino-américaine de cette époque n’est pas en mesure de s'approprier cette technologie et il n'est pas souhaitable qu'elle le fasse. Lart qui en découle est appauvri, car la société technologique développe des «signaux» de communication, qui remplacent le «signe», dans la relation entre signifié et signifiant, ce qui est problématique dans la mesure où «la señal [es] un indicador mecánico, pauperizado, capacitado para marcar un camino a seguir pero impotente para abrir el complejo meollo de una estructura de sentido como es el lenguaje» (Traba 2005: 64). Par conséquent, l'adoption massive de ce style pictural en Amérique latine est, selon elle, un «cheval de Troie». Des résultats dans l'art latino-américain sont visibles immédiatement et cette "colonisation esthétique» met à mal la cartographie originelle de Marta Traba et son système de zones ouvertes et fermées.

Face à cette «satellisation» vis-à-vis des États-Unis (Traba 2005: 143), Marta Traba élabore sa théorie de la résistance: l'Amérique latine doit se protéger de ce transfert de civilisation qui lui impose un art socialement inadapté et esthétiquement stérile. Elle isole, chez certains artistes, de nouvelles propositions esthétiques, où elle identifie des conditions de renaissance de l'art moderne en Amérique latine, sur le plan du style, du médium ou du message des œuvres. Sur le plan du style, elle prend comme modèle les initiatives personnelles d'Obregón ou de Botero, qui sont des artistes modernes colombiens mais ne suivent pas les modèles nord-américains. Botero (1932) subvertit le style du poster, image plane et en deux dimensions dont la fonction est de "montrer" brièvement, en rendant aux sujets représentés le volume qu'ils ont perdu et en donnant à voir l'immobilité, le temps figé qui participent d'une représentation topique colombienne. Par ailleurs, le médium utilisé par l'artiste peut, lui aussi, redonner une signification à l'art. Ceci explique l'important retour au dessin ou à la gravure d'artistes comme José Luis Cuevas, Juan Antonio Roda ou Luisa Palacios. Leur nombre et leur visibilité augmentent considérablement grâce aux diverses biennales (dès 1963) et à l'Exposition Latino-américaine de Dessin et de Gravure de 1967 au Venezuela. C'est le symptôme d'une expression artistique qui retrouve une fonction communicative et se reconnecte avec la société, grâce, notamment, à sa simplicité. Enfin, Marta Traba propose de détourner le message véhiculé par le pop'art. Que la parodie soit humoristique ou subversive, il s'agit alors d'utiliser le pop'art comme un boomerang contre ceux qui l'ont émis, ou de réaliser une «nacionalización del pop» (Traba 2005: 218). Selon Marta Traba, Santiago Cárdenas (1937), par exemple, réussit une appropriation critique et consciente, en décontextualisant des objets et en créant une peinture atemporelle et poétique. 
La représentation rigoureuse des objets ne sert alors plus ni à la production ni à la consommation en masse. Le détournement peut aussi être plus directement subversif: le pop'art devenant ainsi le vecteur d'une critique sociale ou politique, comme le montrent les œuvres de Clemencia Lucena (1945-1983) qui ouvrent la voie d'une libération culturelle, grâce à « un arte que nos sirva de arma contra la alienación" (Clemencia Lucena citée par Traba 1995: 218). Dans le même esprit, l'œuvre de Beatriz González (1938), dans un style pop très personnel, met en lumière La historia extensa de Colombia - expression par laquelle elle désigne l'histoire et les discours officiels fallacieux, et la création des héros nationaux - en la désacralisant et en dénonçant sa fonction idéologique pernicieuse. Elle qualifie elle-même son œuvre, délaissant la société technologique, de "pintura para países subdesarrollados» (Traba 1977: 40). C'est, d'après Marta Traba, à ces conditions que l'art peut retrouver sa capacité à communiquer avec la société qui le voit naître, en questionnant les propositions esthétiques qui viennent de l'extérieur pour trouver des solutions picturales nouvelles et signifiantes.

\section{Conclusion}

L'œuvre critique de Marta Traba n'est pas une simple évaluation esthétique ou une série de propositions théoriques: c'est d'abord un travail global et puissant de mise en crise. Marta Traba a montré où et comment l'histoire de l'art moderne latino-américain prenait ses tournants, elle a contribué à dessiner, l'une après l'autre et l'une par l'autre, ces deux périodes charnières et à en exprimer les formes et les modalités. Puis, dans le cadre d'une théorie artistique d’ampleur, aux fondements historiques, socioculturels, économiques, elle défend de nouvelles pistes, de nouvelles normes. Sans doute est-ce là le point extrême du travail critique, et le plus délicat: chercher à trancher le nœud que l'on a vu se nouer.

La pensée radicale et parfois polémique de Marta Traba a eu un fort impact sur son époque et elle est révélatrice de l'effervescence intellectuelle des années 1960-1970 en Amérique latine. Ses préoccupations identitaires, qui ont marqué la production de nombreux artistes, sont fortement ancrées dans ce contexte, mais elles continuent, aujourd'hui encore, d'infuser dans le débat artistique latino-américain.

\section{Bibliographie}

Bayón, D. (ed.), 1980 [1974], América Latina en sus artes, México, Siglo XXI Editores.

Bazzano, F., González, B., 2006, «¿Qué nos dejó Marta Traba?», Arcadia, 12, p. 22-23.

Belting, H., 2004, Pour une anthropologie des images, Paris, Gallimard.

Giraldo, E., 2007, Marta Traba: crítica del arte latinoamericano, Medellín, La Carreta Editores EU. 
Gómez Echeverri, N., 2008, En blanco y negro. Marta Traba en la televisión colombiana 1954-1958, Bogotá, Ediciones Uniandes.

Mosquera, G. (ed.), 1995, Beyond the fantastic. Contemporary art criticism from Latin America, Londres, IVA.

Osterwold, T., 2011 [1990], Pop Art, Cologne, Taschen.

Pizarro, A., 2002, Las grietas del proceso civilizatorio: Marta Traba en los sesenta, Santiago de Chile, LOM Ediciones.

Rama, A., 1987 [1982], Transculturación narrativa en América Latina, México, Siglo XXI Editores.

Rodríguez Prampolini, I., 1997 [1964], La crítica de arte en México en el siglo XIX, México, UNAM.

Traba, M., 1961, La pintura nueva en Latinoamérica, Bogotá, Ediciones Librería central.

Traba, M., 1972, Arte Latinoamericano actual, Caracas, Ediciones de la Biblioteca de la Universidad Central de Venezuela.

Traba, M., 2005 [1973], Dos décadas vulnerables en las artes plásticas latinoamericanas, 1950-1970, Buenos Aires, Siglo XXI Editores.

Traba, M., 1973, "La pintura como medio de comunicación», in: Araújo de Vallejo E., 1984, Marta Traba, Bogotá, Planeta, p. 295-298.

Traba, M., 1975, La zona del silencio, México, Fondo de Cultura Económica.

Traba, M., 1977, Los muebles de Beatriz González, Bogotá, Museo de Arte Moderno, Carlos Valencia Editores.

Traba, M., 1994, Arte de América latina 1900-1980, Washington, Banco Interamericano de Desarrollo.

Zalamea, G. (ed.), 2010, El programa cultural y político de Marta Traba, Bogotá, Editorial Universidad Nacional de Colombia. 